\title{
Many-objective optimization for coordinated operation of integrated electricity and gas network
}

\author{
Y. N. KOU ${ }^{1}$, J. H. ZHENG ${ }^{1}$, Zhigang $\mathrm{LI}^{1}$, Q. H. WU ${ }^{1,2}$
}

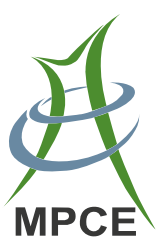

\begin{abstract}
This paper develops a many-objective optimization model, which contains objectives representing the interests of the electricity and gas networks, as well as the distributed district heating and cooling units, to coordinate the benefits of all parties participated in the integrated energy system (IES). In order to solve the many-objective optimization model efficiently, an improved objective reduction (IOR) approach is proposed, aiming at acquiring the smallest set of objectives. The IOR approach utilizes the Spearman's rank correlation coefficient to measure the relationship between objectives based on the Pareto-optimal front captured by the multi-objective group search optimizer with adaptive covariance and Lévy flights algorithm, and adopts various strategies to reduce the number of objectives gradually. Simulation studies are conducted on an IES consisting of a modified IEEE 30-bus electricity network and a 15-node gas network. The results show that the many-objective optimization problem is transformed
\end{abstract}

CrossCheck date: 28 February 2017

Received: 31 December 2016/Accepted: 28 February 2017/Published online: 26 April 2017

(C) The Author(s) 2017. This article is an open access publication

$\bowtie$ J. H. ZHENG

z.jh09@mail.scut.edu.cn

Y. N. KOU

epyn.kou@mail.scut.edu.cn

Zhigang LI

lizg16@scut.edu.cn

Q. H. WU

wuqh@scut.edu.cn

1 School of Electric Power Engineering, South China University of Technology, Guangzhou 510640, China

2 Department of Electrical Engineering and Electronics, The University of Liverpool, Liverpool L69 3GJ, UK into a bi-objective formulation by the IOR. Furthermore, our approach improves the overall quality of dispatch solutions and alleviates the decision making burden.

Keywords Integrated energy system, Gas network, Electricity network, Many-objective optimization, Objective reduction

\section{Introduction}

In recent years, the demand for energy resources is increasing with the development of many sectors in society, such as the modern agriculture and irrigation systems [1]. Due to the low fuel cost, environment friendliness, and the fast response generating capacity of the gas-fired generator [2], an increasing proportion of the total power production is generated by gas-fired generators. According to the World Energy Outlook 2010 [3], the worldwide demand for natural gas in the power sector in 2008 was $4303 \mathrm{TWh}$, and this amount is projected to rise to 7600 TWh by 2035 , leading to the ever-increasing synergies between the gas network and electricity network. More importantly, with the development of distributed renewable resources and district energy demand [4], the interconnections between the gas network and electricity network extend from the gas-fired generators to various types of connectivity nodes, such as distributed heating and cooling loads [5], combined heating and power [6]. For the highly interdependency between the two energy supply networks, the natural gas transmission could have influence on the power transmission from the perspectives of economics and security, and vice versa $[7,8]$. Therefore, it is wise to treat the gas network and electricity network coordinately. 
Extensive research has been conducted on the coordinated planning and operation of the integrated electricity and gas network. A multi-objective optimization for combined gas and electricity network expansion planning was presented in [9]. The objectives were to minimize both investment cost and production cost of the combined system while taking into account the $N-1$ network security criterion. The coordinated scheduling of electricity and gas network was described as a bi-level programming formulation in [10]. The objective of the upper-level problem is to minimize the operating cost of electricity network while the scheduling optimization problem of gas network is embedded within the lower-level problem. In [11], an interval optimization based operating strategy for gaselectricity integrated energy systems was proposed considering demand response and wind uncertainty. In most of the previous research, only the gas-fired generator has been considered as the linkage between the electricity network and gas network. Reference [12] proposed the model of an integrated energy system (IES) with electricity network, gas network, and district heating and cooling units (DHCs) embedded, in which both the gas-fired generators and DHCs are regarded as the interconnections of the electricity network and gas network. Then a coordinate scheduling strategy was utilized to optimize conflicting benefits of the two networks. The simulation results verified that the coordinate scheduling strategy can obtain better solutions of the power generation cost and gas supply cost compared with the single objective optimization method.

Although $[9,12]$ adopted the multi-objective optimization model to tackle the planning and operation problems of the integrated electricity and gas network, only the costs of the two networks were taken into consideration from the economic point of view. The power system dispatch problem minimizing the emissions and fuel cost simultaneously is referred as environmental/economic dispatch (EED), and it has attracted much attention of researchers to settle this issue $[13,14]$. Therefore, the emissions of polluting gases are also worth being optimized from the perspective of environmental protection. In the meanwhile, natural gas transmission could affect the security of power transmission. The optimal dispatch of an IES is modeled as a many-objective problem (MaOP) after taking all the above factors into consideration, which is classified as a special paradigm of multi-objective optimization problem with more than three objectives $[15,16]$.

This paper proposes a many-objective optimization model for the coordinated optimal dispatch of an IES. The proposed model contains objectives representing the interests of the electricity network, the gas network and DHCs, as well as the environmental protection and system security, to coordinate the benefits of different parties.
Although [17] has taken many objectives into consideration, it also only selected two objectives, the costs of the power system and DHCs, for optimization and regarded the rest attributes as the multiple criteria for decision making. Strictly speaking, this handling method dose not belong to the area of many-objective optimization, so the technical distinction between them would be significant.

The existing multi-objective evolutionary algorithms (MOEAs) have shown their capacity in optimizing the problems with few objectives (about three or so), however, their performance reduces exponentially in regard of optimizing the MaOPs [18]. For one thing, the selection of Pareto-based non-dominated solutions becomes ineffective as the majority of the solutions are non-comparable. For another, it would be difficult to visualize a large dimensional Pareto-optimal front and the decision making task would be arduous since there is a large number of nondominated solutions to choose. To overcome the difficulties in tackling MaOPs, the authors in [19] proposed an improved $\alpha$-dominance strategy to improve the discrimination between solutions, but its performance is still not satisfactory. Reference [20] transformed MaOPs into several single-objective optimization problems by aggregation functions with a series of weight vectors, but its performance on MaOPs with highly correlated objectives is modest. An objective reduction approach was proposed in [21] to select the most conflicting objectives during the execution of MOEAs, and simulation results showed that this approach improved the performance of Pareto-based MOEAs significantly. However, this objective reduction approach might be ineffective for MaOPs without redundant objectives. In [22], the concept of aggregation tree was introduced for visualization and dimension reduction in many-objective optimization, and it also presented strategies that the decision maker can use to acquire better solutions. Reference [23] applied the aggregation tree to solve a real-word MaOP, the interior permanent magnet motor design problem, providing directions for an informed choice of objectives in the design problem. The main disadvantage of this approach is that it always chooses the two most harmonious objectives in a greedy fashion, which might not lead to the best combination of objectives.

To obtain an objective set with fewer number and better optimization results, this paper proposes an improved objective reduction (IOR) approach for MaOPs. This approach employs the Spearman's rank correlation coefficient [24] to measure the correlation degrees of objectives, and implements various strategies to reduce the number of objectives. An automatic objective aggregation procedure is first applied to sum up the harmonious objectives into a single new compound objective. Secondly, the objectives whose values vary within small ranges during the 
optimization process are transformed into constraints. Thirdly, the objective that conflicts with other objectives mostly is removed to promote the overall performance. Lastly, the remaining objectives are divided into two conflicting groups based on their Spearman's rank correlation coefficients.

The novelty of this paper lies in that it develops a many-objective optimization model which contains objectives representing the interests of different parties in an IES. Compared with the previous works that only consider two or three objectives, this model has a wider index coverage and is closer to the reality. Furthermore, this paper proposes a novel objective reduction approach named IOR to tackle the many-objective optimization model, in which the relationships among the many objectives are quantitatively analyzed and a specific reduced formulation of the original MaOP can be defined via various strategies.

\section{Integrated energy system modeling}

\subsection{System description}

An IES with electricity network, gas network, and DHCs embedded [12] is illustrated in Fig. 1. The system includes the electricity supply system, electricity transmission system, electricity load, gas supply system, gas transmission system, gas-fired generator, gas load, heating and cooling load. The electricity network and gas network are closely interconnected by the gas-fired generator which can convert natural gas into electricity power for electricity network, and the distributed DHC which consumes both the electricity power and natural gas to serve its heating and cooling load.

The dispatch problem of an IES studied in this paper conducts in 1-hour interval [10-12, 25]. In order to compromise the competing benefits of the electricity network, gas network, and distributed DHCs, the power trade-offs among them are investigated in detail by means of

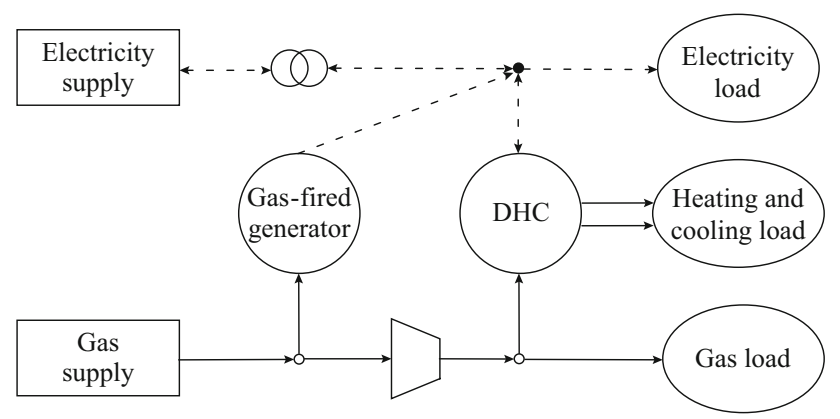

Fig. 1 Framework of an IES with electricity network, gas network and DHCs embedded optimizing the selected objectives simultaneously. The objective of gas network is its operation profit, while the operation cost of DHCs is treated as an objective. Both of these two objectives are selected from the economic viewpoint. As the main network included in the IES, the electricity network chooses the fuel cost, power loss, $\mathrm{NO}_{\mathrm{x}}$ emission, $\mathrm{SO}_{2}$ emission, voltage deviation, and voltage stability as the objectives to represent its economic, environmental, and reliable benefits. Therefore, the dispatch problem of an IES is modeled as a MaOP, which is formulated in detail in the following sections.

\subsection{Modelling of gas network}

Generally, the gas network consists of gas wells, gas pipelines, gas compressors, interconnection points, gas storage stations and gas loads [26]. Natural gas is injected from gas wells and flows through pipelines, and then delivered to the gas loads. The gas compressors are installed to compensate the pressure loss due to the friction of pipelines. The gas storage stations, which provide a buffer to coordinate the usage of gas during multiple periods, are not taken into consideration in this paper.

\subsubsection{Operation profit}

The objective of the gas network is to maximum its operation profit, that is, the total benefit of gas consumption minus the sum of gas production cost, which can be depicted as follows:

$F_{\text {gas }}=\sum_{d} \lambda_{d} Q_{d}-\sum_{w} \lambda_{w} Q_{w}$

where $\lambda_{d}$ and $Q_{d}$ are the gas price and gas consumption of gas load $d ; \lambda_{w}$ and $Q_{w}$ are the gas price and gas production of gas well $w$, respectively.

\subsubsection{Constraints}

At each node of the gas network, the nodal gas flow balance must be satisfied to assure that the sum of the gas injection to a node is equal to zero, that is:

$$
Q_{i}+\sum_{n \in i} f_{n i}-\sum_{m \in i} f_{i m}-\sum_{j} G_{i j} Q_{\mathrm{c}}\left(H_{j}\right)=0
$$

where $Q_{i}$ is the net gas injection at node $i$, including the positive injection of gas suppliers and the negative injection of gas loads; $n \in i$ means that node $n$ is adjacent to node $i, f_{n i}$ is the gas injection from the upstream node $n$, and $f_{i m}$ is the gas output to the downstream node $m ; H_{j}$ represents the horsepower of compressor $j ; Q_{\mathrm{c}}\left(H_{j}\right)$ is the corresponding gas consumption of compressor $j$, which is expressed as: 


$$
Q_{\mathrm{c}}\left(H_{j}\right)=a_{\mathrm{cj}} H_{j}^{2}+b_{\mathrm{c} j} H_{j}+c_{\mathrm{c} j}
$$

where $a_{\mathrm{c} j}, b_{\mathrm{c} j}$ and $c_{\mathrm{c} j}$ are the coefficients of the gas consumption of compressor $j ; G_{i j}=1$ if $Q_{\mathrm{c}}\left(H_{j}\right)$ is supplied by gas node $i$.

In this paper, the gas loads include the gas-fired generators and distributed DHCs, along with the regular gas loads. The gas loads can be regarded as negative gas injections at the gas load nodes. Among them, the gas consumption of the gas-fired unit is determined by its hourly power generation dispatch of the electricity network, which is calculated as [25]:

$$
Q_{\mathrm{GU} i}=\alpha_{i}+\beta_{i} P_{\mathrm{GU} i}+\gamma_{i} P_{\mathrm{GU} i}^{2}
$$

where $\alpha_{i}, \beta_{i}$ and $\gamma_{i}$ are the gas consumption function coefficients of gas-fired unit $i$; $P_{\mathrm{GU} i}$ is the power generation of gas-fired unit $i$, which is treated as a control variable in this paper.

The gas flow from node $m$ to node $n, f_{m n}(\mathrm{kcf} / \mathrm{hr})$ is computed as:

$f_{m n}=\operatorname{sgn}\left(\pi_{m}, \pi_{n}\right) C_{m n} \sqrt{\left|\pi_{m}^{2}-\pi_{n}^{2}\right|}$

where $\pi_{m}$ and $\pi_{n}$ are the pressures at nodes $m$ and $n$ respectively; $\operatorname{sgn}\left(\pi_{m}, \pi_{n}\right)=1$ if $\left(\pi_{m}^{2}-\pi_{n}^{2}\right)>0$ and $\operatorname{sgn}\left(\pi_{m}, \pi_{n}\right)=-1$ if $\left(\pi_{m}^{2}-\pi_{n}^{2}\right)<0 ; C_{m n}$ is a constant related to the physical characteristic of each pipeline, which is given by:

$$
C_{m n}=3.2387 \frac{T_{0}}{\pi_{0}} \sqrt{\frac{D_{m n}^{5}}{L_{m n} G Z^{\mathrm{a}} T_{m n}^{\mathrm{a}} F_{m n}}}
$$

where $T_{0}$ is the standard temperature, $520^{\circ} \mathrm{R} ; \pi_{0}$ is the base pressure, $14.65 \mathrm{psia} ; D_{m n}$ is the inner diameter of the pipeline between nodes $m$ and $n$, inch; $L_{m n}$ is the length of pipeline between nodes $m$ and $n$, mile; $G$ is the gas specific gravity, $0.6 ; Z^{\mathrm{a}}$ is the average gas compressibility factor; $T_{m n}^{\mathrm{a}}$ is the average gas temperature; $F_{m n}$ is the friction factor of the pipeline and can be given as a function of $D_{m n}$ [27]:

$$
F_{m n}=\frac{0.032}{D_{m n}^{1 / 3}}
$$

During the long transmission distance of gas in pipe-lines, the gas compressors are installed to compensate the loss of pressure due to the friction of pipelines. The gas flow from node $m$ to node $n$ through the compressor $j, f_{m n}$, is mathematically expressed as [11]:

$f_{m n}=\operatorname{sgn}\left(\pi_{m}, \pi_{n}\right) \frac{H_{j}}{k_{2 j}-k_{1 j} R_{j}^{\alpha}}$

where $k_{1 j}, k_{2 j}, \alpha$ are empirical parameters related to the compressor characteristics; $R_{j}=\frac{\max \left(\pi_{m}, \pi_{n}\right)}{\min \left(\pi_{m}, \pi_{n}\right)}$ is the compression ratio of compressor $j$.
The inequality constraints of gas network are derived from the limits of gas wells' capacities, the compressors' horsepower, compression ratios, and the nodal pressure constraints, which are summarized as:

$$
\begin{aligned}
& Q_{w}^{\min } \leq Q_{w} \leq Q_{w}^{\max } \\
& H_{j}^{\min } \leq H_{j} \leq H_{j}^{\max } \\
& R_{j}^{\min } \leq R_{j} \leq R_{j}^{\max } \\
& \pi_{k}^{\min } \leq \pi_{k} \leq \pi_{k}^{\max }
\end{aligned}
$$

\subsection{Modelling of DHC}

A schematic diagram of the DHC unit [17] is illustrated in Fig. 2. As shown in the figure, the DHC unit consists of four kinds of energy resources: electricity power, wind power, natural gas, and solar radiation, which are utilized by the energy conversion facilities to serve the heating and cooling loads located in a residential area.

The optimal operation of the DHC refers to the minimization of the operation cost which consists of the cost of purchasing electric power from the electricity network and purchasing natural gas from the gas network. In this paper, the power trade-off $P_{\text {ele }}$ between the electricity network and the DHC is regarded as a control variable.

\subsubsection{Operation cost}

As stated previously, the objective of DHCs is to minimize the total operation cost, which can be depicted as follows:

$$
\begin{aligned}
F_{\mathrm{dhc}} & =\sum_{i}^{N_{\mathrm{DHC}}}\left(C_{\mathrm{ele}_{i}}+C_{\mathrm{g}_{i}}\right) \\
& =\sum_{i}^{N_{\mathrm{DHC}}}\left(1000 c_{\mathrm{ele}_{\mathrm{e}}} P_{\mathrm{ele}_{i}}+3600 c_{\mathrm{g}} B_{\mathrm{g}_{i}}\right)
\end{aligned}
$$

where $N_{\mathrm{DHC}}$ is the number of DHCs; $c_{\text {ele }}(\$ / \mathrm{kWh})$ is the electricity tariff rate of purchasing power from the electricity network $P_{\text {ele }}(\mathrm{MW}) ; c_{\mathrm{g}}\left(\$ / \mathrm{m}^{3}\right)$ is the unit price of

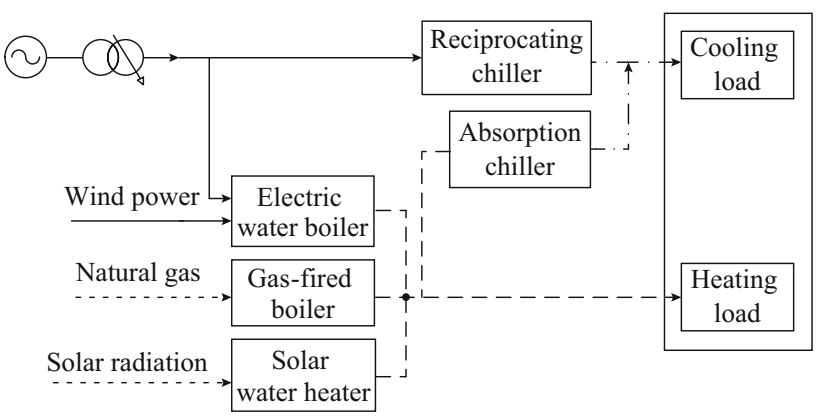

Fig. 2 Schematic diagram of DHC unit 
natural gas $B_{\mathrm{g}}\left(\mathrm{m}^{3} / \mathrm{s}\right)$. The coefficient '1000' and '3600' in (13) is caused by the unit conversion.

\subsubsection{Constraints}

The operating constraints of the DHCs include equality and inequality constraints. The equality constraint, listed in (14) is derived from power balance.

$$
P_{\mathrm{h} 1}+P_{\mathrm{h} 2}+P_{\mathrm{h} 3}+P_{\mathrm{c} 1}+P_{\mathrm{c} 2}=P_{\mathrm{load}}
$$

where $P_{\mathrm{h} 1}$ is the sum of the electricity power and wind power, $\eta_{1}\left(P_{\text {ele }}+P_{\mathrm{WG}}\right) ; P_{\mathrm{h} 2}$ is equal to $\eta_{2} q_{\mathrm{g}} B_{\mathrm{g}} ; P_{\mathrm{h} 3}$ is the output of the solar water heater which converts its power input $N_{\text {col }} A_{\mathrm{c}} H_{\mathrm{T}}$ into heat production with the efficiency rate $\eta_{\mathrm{c}}$ of the solar collector; $P_{\mathrm{c} 1}$ is the cooling output of the reciprocating chiller, while $P_{\mathrm{c} 2}$ is the cooling output of the absorption chiller. In addition, $P_{\text {load }}$ is the sum of heating loads and cooling loads.

The inequality constraints are derived from the limits of heat unit capacity, heat charge, discharge rate, and the limits of chillers' cooling capacities, which are summarized as:

$$
\begin{aligned}
& P_{\mathrm{h} 1}^{\min } \leq P_{\mathrm{h} 1} \leq P_{\mathrm{h} 1}^{\max } \\
& P_{\mathrm{h} 2}^{\min } \leq P_{\mathrm{h} 2} \leq P_{\mathrm{h} 2}^{\max } \\
& P_{\mathrm{h} 3}^{\min } \leq P_{\mathrm{h} 3} \leq P_{\mathrm{h} 3}^{\max } \\
& P_{\mathrm{c} 1}^{\min } \leq P_{\mathrm{c} 1} \leq P_{\mathrm{c} 1}^{\max } \\
& P_{\mathrm{c} 2}^{\min } \leq P_{\mathrm{c} 2} \leq P_{\mathrm{c} 2}^{\max }
\end{aligned}
$$

\subsection{Modelling of electricity network}

The optimal operation of the electricity network plays a significant role because the electricity network is the main network included in the IES. The optimal operation is concerned with optimizing the generation dispatch of the distributed generators, the real power loss of each transmission line, the voltage stability of each node, and the power quality of each bus. In addition, the operation must satisfy a set of constraints. The corresponding objectives and constraints formulated for the model of the electricity network are described in the following sections.

\subsubsection{Fuel cost}

The total fuel cost is related to the power outputs of distributed generators, which can be mathematically modelled as a quadratic function [13]:

$$
F_{\mathrm{ele} 1}=\sum_{i}^{N_{\mathrm{G}}} F_{i}=\sum_{i}^{N_{\mathrm{G}}}\left(a_{i} P_{\mathrm{G}_{i}}^{2}+b_{i} P_{\mathrm{G}_{i}}+c_{i}\right)
$$

where $N_{\mathrm{G}}$ is the number of generators; $F_{i}$ is the fuel cost of generator $i ; a_{i}, b_{i}$, and $c_{i}$ are the corresponding coefficients.

\subsubsection{Power loss}

The power loss is inevitable when power flowing on the transmission lines, and it causes economic lose. The sum of power losses can be calculated by the following equation [13]:

$F_{\mathrm{ele} 2}=\sum_{k}^{N_{\mathrm{TL}}} g_{k}\left[V_{i}^{2}+V_{j}^{2}-2 V_{i} V_{j} \cos \left(\delta_{i}-\delta_{j}\right)\right]$

where $N_{\mathrm{TL}}$ is the number of transmission lines, $g_{k}$ is the conductance of $k$ th line connected between $i$ th and $j$ th bus, $V_{i}, V_{j}, \delta_{i}, \delta_{j}$ are the voltage magnitudes and phase angles of the $i$ th and $j$ th bus, respectively.

\subsection{3 $\mathrm{NO}_{x}$ emission}

The emission of $\mathrm{NO}_{x}$ in (ton/h) is a function of the generator outputs, i.e., the summation of a quadratic function and an exponential function [13]:

$$
F_{\text {ele } 3}=\sum_{i}^{N_{\mathrm{G}}} 10^{-2}\left(\tau_{0 i}+\tau_{1 i} P_{\mathrm{G}_{i}}+\tau_{2 i} P_{\mathrm{G}_{i}}^{2}+\epsilon_{i} e^{\xi_{i} P_{\mathrm{G}_{i}}}\right)
$$

where $\tau_{0 i}, \tau_{1 i}, \tau_{2 i}, \epsilon_{i}$, and $\xi_{i}$ are the coefficients of the $i$ th generator $\mathrm{NO}_{x}$ emission characteristics.

\subsection{4 $\mathrm{SO}_{2}$ emission}

The emission of $\mathrm{SO}_{2}$ in (ton/h) is assumed to be a quadratic polynomial function of the power outputs of generators [14]:

$$
F_{\text {ele } 4}=\sum_{i}^{N_{\mathrm{G}}} 10^{-2}\left(\tau_{3 i}+\tau_{4 i} P_{\mathrm{G}_{i}}+\tau_{5 i} P_{\mathrm{G}_{i}}^{2}\right)
$$

where $\tau_{3 i}, \tau_{4 i}, \tau_{5 i}$ are the coefficients of the $i$ th generator $\mathrm{SO}_{2}$ emission characteristics.

\subsubsection{Voltage deviation}

As bus voltage is one of the most important power quality and security indices for electricity network operation, the sum of the voltage deviations between each load bus voltage magnitude $V_{j}$ and the referenced voltage magnitude $V_{\text {ref }}$ is usually treated as an objective [28]:

$F_{\text {ele } 5}=\sum_{j}^{N_{\mathrm{B}}}\left|V_{j}-V_{\text {ref }}\right|$ 


\subsubsection{Voltage stability}

This objective is to maintain the voltage stability and move the system far away from the voltage collapse point. This can be achieved by minimizing the maximum voltage stability indicator and this objective can be expressed as [17]:

$F_{\text {ele6 }}=\max _{j \in N_{\mathrm{L}}}\left(1-\sum_{i}^{N_{\mathrm{G}}} F_{j i} \frac{V_{i}}{V_{j}}\right)$

where matrix $\boldsymbol{F}$ is depicted by:

$\boldsymbol{F}=-\boldsymbol{Y}_{\mathrm{LL}}^{-1} \boldsymbol{Y}_{\mathrm{LG}}$

where $\boldsymbol{Y}_{\mathrm{LL}}$ and $\boldsymbol{Y}_{\mathrm{LG}}$ are the submatrices of the Jacobian matrix. $\boldsymbol{Y}_{\mathrm{LL}}$ consists of the admittance elements between load nodes while $\boldsymbol{Y}_{\mathrm{LG}}$ consists of those between load nodes and generator nodes.

\subsubsection{Constraints}

The optimal operation of the electricity network must satisfy a variety of constraints from the viewpoint of power balance and reliable operation. The equality constraints are the balance limits of active and reactive power described by a set of non-linear power flow equations as follows:

$$
\begin{gathered}
P_{\mathrm{G}_{i}}-P_{\mathrm{D}_{i}}-V_{i} \sum_{j=1}^{N} V_{j}\left(G_{i j} \cos \theta_{i j}+B_{i j} \sin \theta_{i j}\right)=0 \\
Q_{\mathrm{G}_{i}}-Q_{\mathrm{D}_{i}}-V_{i} \sum_{j=1}^{N} V_{j}\left(G_{i j} \sin \theta_{i j}-B_{i j} \cos \theta_{i j}\right)=0
\end{gathered}
$$

Apart from equality constraints, there is also a set of inequality constraints associated with the distributed generators, transformers, shunt compensators, and transmission lines, respectively. The corresponding limits of active power, reactive power, and voltage of generators are given by:

$P_{\mathrm{G}_{i}}^{\min } \leq P_{\mathrm{G}_{i}} \leq P_{\mathrm{G}_{i}}^{\max }$

$Q_{\mathrm{G}_{i}}^{\min } \leq Q_{\mathrm{G}_{i}} \leq Q_{\mathrm{G}_{i}}^{\max }$

$V_{\mathrm{G}_{i}}^{\min } \leq V_{\mathrm{G}_{i}} \leq V_{\mathrm{G}_{i}}^{\max }$

In addition, the constraints of transformers, shunt compensators, and transmission lines are given as follows:

$$
\begin{aligned}
& T_{m}^{\min } \leq T_{m} \leq T_{m}^{\max } \\
& Q_{\mathrm{C}_{n}}^{\min } \leq Q_{\mathrm{C}_{n}} \leq Q_{\mathrm{C}_{n}}^{\max } \\
& V_{\mathrm{L}_{i}}^{\min } \leq V_{\mathrm{L}_{i}} \leq V_{\mathrm{L}_{i}}^{\max } \\
& \left|S_{\mathrm{L}_{i}}\right| \leq S_{\mathrm{L}_{i}}^{\max }
\end{aligned}
$$

\section{Optimal coordinated operation of IES}

The optimal coordinated operation of an IES consisting of gas network and distributed DHCs interconnected via the electricity network is formulated as a MaOP mathematically. As mentioned in above section, the objective function of the many-objective optimization model formulated for the IES considering the benefits of all parties is given by:

$\min \left(-F_{\text {gas }}, F_{\text {dhc }}, F_{\text {ele } 1}, F_{\text {ele } 2}, F_{\text {ele3 }}, F_{\text {ele } 4}, F_{\text {ele } 5}, F_{\text {ele } 6}\right)$

where the operation profit of gas network $F_{\text {gas }}$ is converted into $-F_{\text {gas }}$ since it is a maximum optimization problem.

In this paper, we suppose the electricity network and gas network are held by different companies. Therefore, although $-F_{\text {gas }}, F_{\text {dhc }}$ and $F_{\text {elel }}$ all belong to economical objectives, they are treated as separate objectives since they stand for the benefits of different parties. And the three objectives are normalized to keep the economical objectives in basically the same number scale. Furthermore, the fuel cost $F_{\text {ele1 }}$ and power loss $F_{\text {ele2 }}$ are also treated separately for the following two reasons. For one thing, the multi-objective optimization of optimal power flow has treated the fuel cost and power loss separately to optimize the power system comprehensively and obtain the compromise solutions, according to [13, 29]. For the other, although the two objectives are both economic factors, they might represent the benefits of different companies. For example, in China the power station concerns the minimization of fuel cost whereas the power grid company wants to minimize the power loss. Therefore, it is more reasonable to formulate these two objectives separately to coordinate the interests of different parties.

Moreover, the optimization problem must satisfy various of constraints aforementioned to maintain the reliable operation of the IES, which are summarized as follows:

1) Gas network constraints: (2)-(12).

2) DHCs constraints: (14)-(19).

3) Electricity network constraints: (27)-(35).

The IES dispatch problem formulated is a complex $\mathrm{MaOP}$ addressed with inequality constraints and non-linear equality constraints. In [12, 17], the efficiency of multiobjective group search optimizer with adaptive covariance and Lévy flights (MGSO-ACL) algorithm has been verified for the multi-objective optimization problems with two objectives. However, the MGSO-ACL has not been tested on problems with more than three objectives, that is, the MaOPs. To further investigate its performance, the MGSOACL is employed to tackle the many-objective optimization model in this paper. 


\subsection{Many-objective optimization}

\subsubsection{Multi-objective group search optimizer with adaptive covariance and Lévy flights}

The individuals of MGSO-ACL are classified into three types, the producer, scrounger, and ranger, based on their fitness values. The number of producers is equal to the number of objectives $\left(N_{\mathrm{ob}}\right)$, which means each producer is assigned to find the best fitness value $F_{p}\left(x_{p}^{(g)}\right),(p=$ $\left.1,2, \cdots, N_{\mathrm{ob}}\right)$ of its corresponding objective, and a number of individuals are randomly selected as the scroungers, then the rest of individuals are named as the rangers. The producers perform the crappie search behavior which is characterized by maximum pursuit angle, maximum pursuit distance, and maximum pursuit height, in the meantime, the producers share the same scroungers and rangers to improve the search efficiency. The main improvements of MGSO-ACL compared with group search optimizer with multiple producers (GSOMP) in [14] lie in the modified searching strategies of scroungers and rangers. In the adopted MGSO-ACL, the adaptive covariance matrix [30] obtained by cumulatively learning for the information organized from the group members of each generation is employed to get a reliably estimator for determining the evolution path and step size for scroungers' behaviors, and Lévy flights [31] are introduced as rangers' searching technique rather than the random walks. For detailed explanations of its searching mechanism, please refer to our previous work [17].

\subsubsection{Pareto-dominance principle}

The Pareto-dominance principle is adopted to obtain the Pareto-optimal solution set which contains a set of optimal non-dominated solutions. The vector $X_{1}$ dominates $X_{2}$ if

$$
\left\{\begin{array}{l}
\forall i, F_{i}\left(X_{1}\right) \leq F_{i}\left(X_{2}\right) \\
\exists j, F_{i}\left(X_{1}\right)<F_{i}\left(X_{2}\right)
\end{array}\right.
$$

The principle is applied by the MGSO-ACL algorithm to select Pareto-optimal solutions in its population at the end of evolution in each iteration, and the Pareto-optimal solutions found during the evolutionary process are stored in an external elitist archive which is updated in every generation. The set of objective value vectors corresponding to the Pareto-optimal solution set is called the Pareto-optimal front.

\subsection{Objective reduction for MaOPs}

If the eight objectives in the many-objective optimization model of the IES are optimized simultaneously, the number of Pareto-optimal solutions will be numerous, which leads to a heavy burden for the optimization and decision making process. Therefore, it is necessary to analyze the relationships among objectives and take measures to reduce the number of objectives.

\subsubsection{Relationship between two objectives}

Generally speaking, when two objectives conflict with each other, it means that the two objectives cannot be improved at the same time. Good values for one implies bad values for the other. The conflict might be global or local, linear or nonlinear. The conflicting objectives are considered to be negatively correlated mathematically.

On the contrary, a harmony relationship between two objectives means that the improvement of one objective would result in an improvement of the other. Harmonious objectives are denoted in parallel coordinate graphs by noncrossing lines and are considered to be positively correlated mathematically. Therefore, the harmonious objectives can be grouped into a single new compound objective through the simple summation without deforming the shape of Pareto-optimal front.

Also, there exists the situation where two objectives are not correlated to each other, that is, they have an independence relationship. As a matter of fact, the degree of conflict or harmony between two objectives can be measured by some methods. The following section introduces a nonparametric correlation computation method using Spearman's rank correlation coefficient.

\subsubsection{Spearman's rank correlation coefficient}

In statistics, Spearman's rank correlation coefficient or Spearman's rho, named after Charles Spearman and often denoted by the Greek letter $\rho$, is a nonparametric measure of statistical dependence between two variables. It assesses how well the relationship between two variables can be described using a monotonic function. If there are no repeated data values, a perfect Spearman correlation of +1 or -1 occurs when each of the variables is a perfect monotone function of the other.

The Spearman correlation coefficient is defined as the Pearson correlation coefficient between the ranked variables. Let $X_{i j}$ be the value for objective $j$ in the solution $i$, then the mathematical formulation of the Spearman's rank correlation coefficient $\rho_{a b}$ between objectives $a$ and $b$ is given below:

$\left\{\begin{array}{l}\rho_{a b}=1-\frac{6 \sum d_{i}^{2}}{n\left(n^{2}-1\right)} \\ d_{i}=K_{i a}-K_{i b}\end{array}\right.$ 
where $n$ is the number of solutions; $d_{i}$ is the difference between rank $K_{i a}$ and $K_{i b}$, and $K_{i j}$ is the rank of $X_{i j}$ within the solutions of objective $j$. Identical values (rank ties or value duplicates) are assigned a rank equal to the average of their positions in the ascending order of the values. Based on the Spearman correlation coefficient matrix $R^{N}=\left\{\rho_{a b}\right\},(1 \leq a \leq N, 1 \leq b \leq N)$, the relations among $N$ objectives can be analyzed.

The sign of the Spearman correlation coefficient $\rho_{a b}$ indicates whether two objectives $a$ and $b$ are in conflict, while the magnitude of $\rho_{a b}$ describes the degree of correlation. If $\rho_{a b}$ is positive with a large value, the two objective are highly positively correlated, i.e., the relationship between $a$ and $b$ is harmony. If $\rho_{a b}$ is negative with a large absolution value, objectives $a$ and $b$ are strongly conflicted. If $\rho_{a b}$ is around zero, it indicates that the two objectives are independent of each other.

Although this nonparametric correlation measure involves loss of information since it ignores the specific values of the objectives, it owes advantages over other measures. First, it works without the requirement of the comparability between objectives and the objectives can use different units without any conversion. Second, it is useful when we do not know the relative importance of each objective. Third, it is robust and insensitive to any previous normalization which would not change the original relationship of the objectives. Fourth, the correlation coefficient is easy to compute and can give a clear description of the relationship between two objectives.

\subsubsection{Procedure of IOR approach}

For the purpose of improving the quality of solutions and reducing the computation burden of decision making, an IOR approach is proposed in this section, as illustrated in Fig. 3.

The first step of our approach is to optimize the original objective set and aggregate the harmonious objectives into a new compound objective through the automatical summation. The pseudo code of the objective aggregation procedure is listed in Table 1. The obtained objective set only consists of relatively conflicting objectives and owes a lower dimension compared with the original problem, which would benefit the optimization process without deforming the shape of Pareto-optimal front.

During the optimization process, there exist objectives whose values vary within small ranges, which means they are wasting the computing resource of MOEAs. In this paper, the coefficient of variation (CV) is adopted to measure the variation degree of each objective. The CV is a standardized measure of dispersion of the data, which is

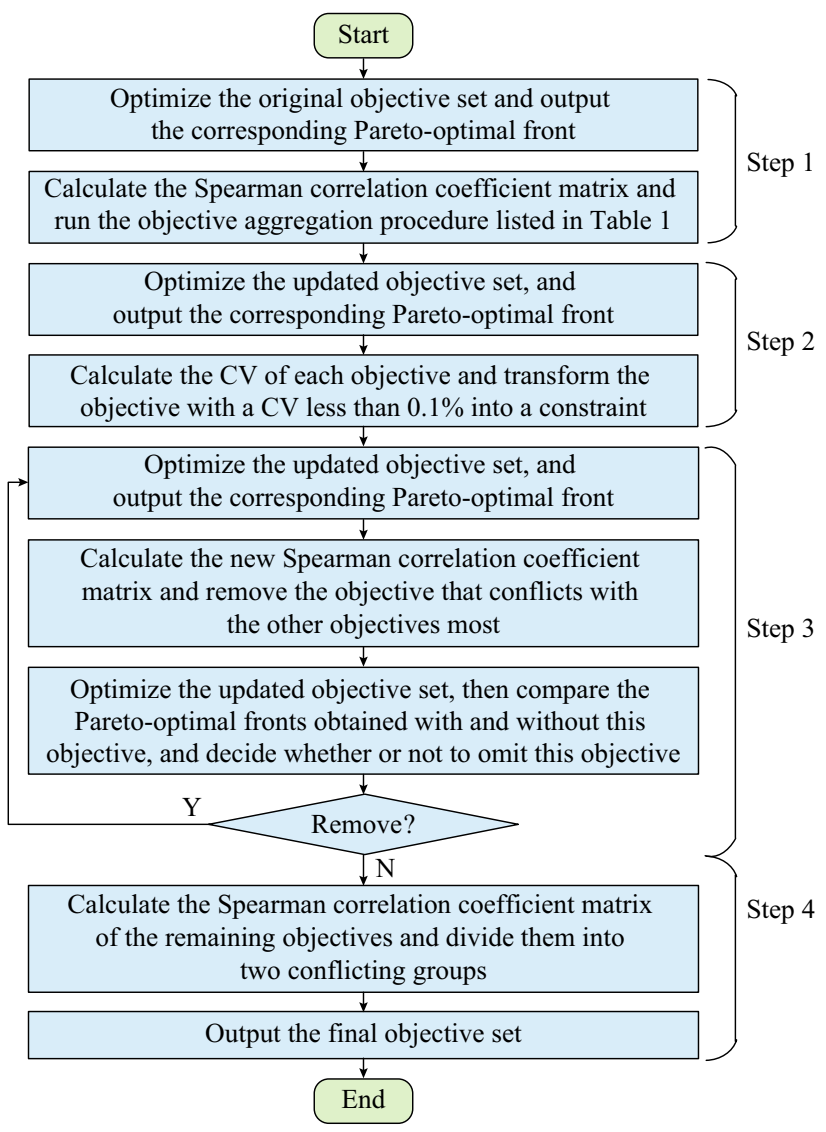

Fig. 3 Framework of IOR approach

defined as the ratio of standard deviation (STD) $\sigma$ to the absolution value of mean $|\mu|$, that is:

$c_{\mathrm{v}}=\frac{\sigma}{|\mu|} \times 100 \%$

Then in the second step, the objective with a small CV, for instance, $0.1 \%$, is transformed into a constraint.

The new Spearman correlation coefficient matrix can be calculated after optimizing the updated problem formulation obtained in the second step. Based on the resultant coefficient matrix, removing the objective that conflicts with the other objectives most is carried out as the third step. This procedure sacrifices the most conflicting objective for the improvement of other objectives, therefore, the decision maker can compare the Pareto-optimal fronts obtained with and without this objective, and then decide whether or not to omit this objective. Furthermore, this step can be repeated until no objective can be removed anymore.

The final step is to divide the remaining objectives into two conflicting groups based on their newly gained Spearman rank correlation coefficients. Accordingly, the final bi-objective formulation can be optimized by the MGSO-ACL algorithm or other MOEAs, and then the 
Table 1 Pseudo code of objective aggregation procedure

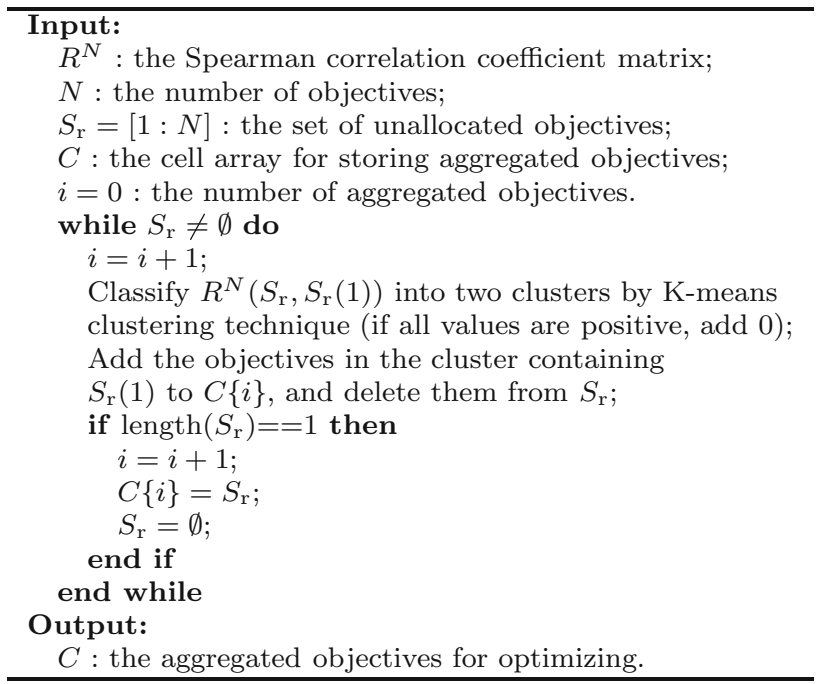

decision maker can select an appropriate solution from the bi-dimensional Pareto-optimal front.

From the above steps, it can be seen that the implementation of the IOR approach depends on the information embedded in the Pareto-optimal front obtained by the MGSO-ACL algorithm, indicating the importance of optimization and optimized values.

\section{Simulation studies}

\subsection{System description}

In this section, simulation studies are conducted on an IES consisting of a modified IEEE 30-bus system, a 15-node gas network, and three distributed DHCs to investigate the proposed many-objective optimization method. The detailed locations of distributed generators, electricity loads, gas fired units and distributed DHCs in the modified IEEE 30-bus system are summarized in Table 2 .

The single-line diagram of the 15-node gas network adopted in this paper is shown in Fig. 4. The gas network consists of 15 nodes, which contains two gas source nodes $(1,2)$, two gas load nodes $(3,4)$, two nodes $(8,9)$ connected

Table 2 Locations of different components in modified IEEE 30-bus system

\begin{tabular}{ll}
\hline Components & Node \\
\hline Generators & $1,2,5,8,13$ \\
Gas-fired units & 7,12 \\
Distributed DHCs & $11,21,30$ \\
Electricity loads & Others \\
\hline
\end{tabular}

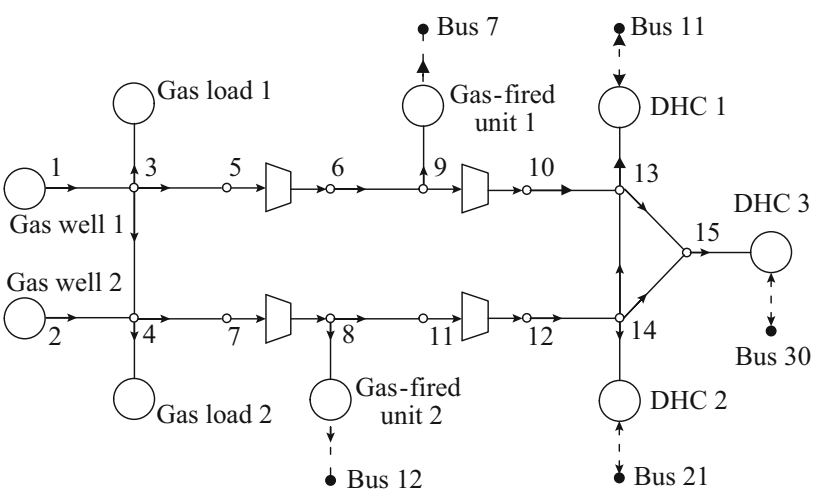

Fig. 4 Single-line diagram of 15-node gas network coupled with IEEE 30-bus network and DHCs

to gas-fired units, and three nodes $(13,14,15)$ connected to distributed DHCs. The gas-fired units serve as gas loads in the gas network and the power sources of the electricity network. The distributed DHCs also serve as gas loads in the gas network, and they also purchase power from the electricity network. In addition, the system parameters and prices are set according to $[5,27,32]$.

\subsection{Many-objective optimization and objective reduction results}

Based on the proposed many-objective optimization model, the MGSO-ACL algorithm and the IOR approach are utilized to obtain the optimal dispatch scheme for the test IES in this paper. The population of individuals and maximum iteration of the MGSO-ACL algorithm are set to be 50 and 200, respectively. Firstly, the eight objectives in (36) are optimized simultaneously to get the MaOP's original Pareto-optimal front which is visualized on parallel coordinate plot in black lines shown in Fig. 5. In parallel coordinate plot, each connected line of the Paretooptimal front represents the values of the eight objectives corresponding to a certain Pareto-optimal solution. According to the Pareto-dominance principle, the Paretooptimal solutions are all optimal non-dominated solutions, and they can achieve the trade-offs in the eight objectives. When many lines cross between two adjacent objectives, it indicates that the two objectives are in a conflicting relationship.

To quantify the correlation degrees among these objectives, the Spearman correlation coefficient matrix, $R^{N}$, is generated based on the objective values corresponding to the Pareto-optimal solutions, as shown in Table 3. From this table, it can be seen that the operation profit of gas network and the operation cost of DHCs are in conflict with each other, the fuel cost, power loss and $\mathrm{NO}_{x}$ emission of electricity network are in harmony with each other, while 


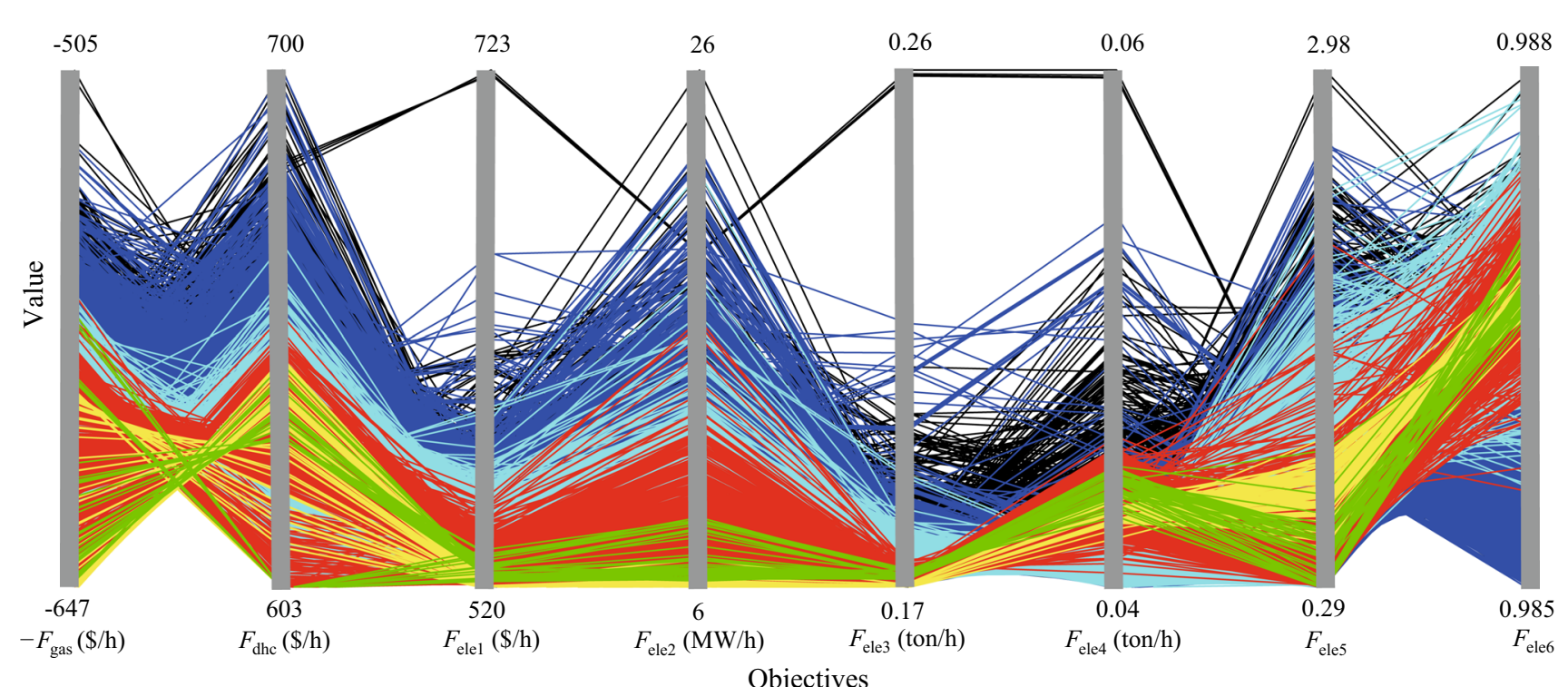

Fig. 5 Pareto-optimal fronts obtained by MGSO-ACL with different objective sets

Table 3 Spearman correlation coefficient matrix calculated with Pareto-optimal solutions of (36)

\begin{tabular}{llllllll}
\hline Objective & $-F_{\text {gas }}$ & $F_{\text {dhc }}$ & $F_{\text {ele1 }}$ & $F_{\text {ele2 }}$ & $F_{\text {ele3 }}$ & $F_{\text {ele4 }}$ & $F_{\text {ele5 }}$ \\
\hline$-F_{\text {gas }}$ & 1 & -0.75 & 0.02 & -0.02 & 0.02 & 0.02 & -0.01 \\
$F_{\text {dhc }}$ & -0.75 & 1 & -0.17 & -0.09 & -0.14 & 0.04 & -0.03 \\
$F_{\text {ele1 }}$ & 0.02 & -0.17 & 1 & 0.75 & 0.89 & -0.44 & -0.13 \\
$F_{\text {ele2 }}$ & -0.02 & -0.09 & 0.75 & 1 & 0.44 & -0.35 & -0.15 \\
$F_{\text {ele3 }}$ & 0.02 & -0.14 & 0.89 & 0.44 & 1 & -0.39 & -0.10 \\
$F_{\text {ele4 }}$ & 0.02 & 0.04 & -0.44 & -0.35 & -0.39 & 1 & -0.22 \\
$F_{\text {ele5 }}$ & -0.01 & -0.03 & -0.13 & -0.15 & -0.10 & -0.10 & -0.10 \\
$F_{\text {ele6 }}$ & -0.08 & 0.04 & -0.22 & -0.30 & -0.14 & 0.01 & 0.01 \\
\hline
\end{tabular}

the other objectives have the relative independence relationships. It is worth mentioning that as the cost of gasfired units is not included in the fuel cost $F_{\text {elel }}$, the interconnection of gas-fired units tends to make $-F_{\text {gas }}$ and $F_{\text {ele } 1}$ positively correlated. However, the coupling of DHCs between the gas and electricity networks complicates the relationship of the two networks. From Table 3, the gas and electricity networks tend to have an independence relationship with the interconnections of gas-fired units and DHCs.

\subsubsection{Automatic objective aggregation}

Based on the obtained Spearman correlation coefficient matrix $R^{N}$ in Table 3, the objective aggregation procedure outputs the objective set to be optimized with the new formulation

$\min \left(-F_{\text {gas }}, F_{\text {dhc }}, F_{\text {a }}, F_{\text {ele } 4}, F_{\text {ele5 }}, F_{\text {ele } 6}\right)$ where $F_{\mathrm{a}}=F_{\text {ele } 1}+F_{\text {ele } 2}+F_{\text {ele3 }}$ is the aggregated objective by the objective aggregation procedure since the three objectives are in harmony with each other.

Then the above objective set is optimized with the MGSO-ACL, and the values of each objective corresponding to the obtained Pareto-optimal solutions is presented in the Pareto-optimal front plotted in Fig. 5 with dark blue lines. By comparing this Pareto-optimal front with the original one, we can see that the loss of quality in Pareto-optimal front of the aggregated formulation is very small, which verifies the effectiveness of summing up the harmonious objectives in dimension reduction for manyobjective optimization.

\subsubsection{Transforming certain objectives into constraints}

Based on the optimization results of (40), the means, STDs, and CVs of the six objectives are listed in Table 4. 
Table 4 Means, STDs and CVs of six objectives based on the optimization result of (40)

\begin{tabular}{llll}
\hline Objectives & Mean & STD & CV $(\%)$ \\
\hline$-F_{\text {gas }}$ & -599.0203 & 26.1270 & 4.36 \\
$F_{\text {dhc }}$ & 632.6249 & 18.2773 & 2.89 \\
$F_{\text {a }}$ & 552.4578 & 14.6585 & 2.65 \\
$F_{\text {ele4 }}$ & 0.0437 & 0.0012 & 2.64 \\
$F_{\text {ele5 }}$ & 0.7622 & 0.3630 & 47.62 \\
$F_{\text {ele6 }}$ & 0.9856 & 0.0005 & 0.05 \\
\hline
\end{tabular}

From this table, the $\mathrm{CV}$ of voltage stability index $\left(F_{\text {ele }}\right)$ is only $0.05 \%$, indicating that this objective nearly stays as a constant during the optimization process. Then it can be treated as a constraint $F_{\text {ele } 6} \leq 1$, to meet the requirement of system stable conditions. Hence, a new problem formulation is given by:

$$
\begin{aligned}
& \min \left(-F_{\text {gas }}, F_{\text {dhc }}, F_{\mathrm{a}}, F_{\text {ele } 4}, F_{\text {ele5 }}\right) \\
& \text { s.t. } F_{\text {ele } 6} \leq 1
\end{aligned}
$$

Solving this new model in (41) leads to the Pareto-optimal front plotted in Fig. 5 with light blue lines. It can be seen that except for the objective $F_{\text {ele6 }}$ which has been turned into a constraint, the other objective values trend to concentrate in the bottom of the figure. This demonstrates that the tiny sacrifice of certain objective will benefit the other objectives a lot, which is meaningful for the many-objective optimization.

\subsubsection{Removing most conflicting objective}

To further reduce the number of objectives to be optimized, we can remove the objective that conflicts with other objectives mostly. By summing up the Spearman correlation coefficients in each column except for the last one of Table 5, the correlation degrees of these objectives are listed in the bottom of the table. It can be seen that the
$\mathrm{SO}_{2}$ emission objective $F_{\text {ele4 }}$ is the most conflicting objective since it has the largest negative value of the correlation degree. Therefore, removing $F_{\text {ele4 }}$ would benefit the optimization of other objectives. The new problem formulation is given by:

$$
\begin{aligned}
& \min \left(-F_{\text {gas }}, F_{\text {dhc }}, F_{\mathrm{a}}, F_{\text {ele } 5}\right) \\
& \text { s.t. } F_{\text {ele } 6} \leq 1
\end{aligned}
$$

The resultant Pareto-optimal front obtained by (42) is projected into the original eight-objective space in Fig. 5 with red lines. From this figure, it can be observed that all the objective values apart from those of $F_{\text {ele } 4}$ are reduced, while the increase of objective values of $F_{\text {ele }}$ is acceptable although it is not being optimized.

\subsubsection{Optimizing two conflicting groups of objectives}

In this section, we make further efforts on reducing objective number and facilitating the decision making task. In order to analyze the relationships among the remaining objectives in (42), the Spearman correlation coefficient matrix of these four objectives is listed in Table 6 based on the Pareto-optimal solutions of (42). As shown in this table, $-F_{\text {gas }}$ and $F_{\text {dhc }}$ have a high conflict degree, while the conflict between $F_{\mathrm{a}}$ and $F_{\text {ele } 5}$ is also relatively high. However, the relationships between other objectives are rather independent. Therefore, there are two versions to

Table 6 Spearman correlation coefficient matrix of four objectives in (42) based on its Pareto-optimal solutions

\begin{tabular}{lllll}
\hline Objective & $-F_{\text {gas }}$ & $F_{\text {dhc }}$ & $F_{\text {a }}$ & $F_{\text {ele5 }}$ \\
\hline$-F_{\text {gas }}$ & 1 & -0.95 & -0.02 & -0.08 \\
$F_{\text {dhc }}$ & -0.95 & 1 & -0.02 & -0.02 \\
$F_{\text {a }}$ & -0.02 & -0.02 & 1 & -0.53 \\
$F_{\text {ele5 }}$ & -0.08 & -0.02 & -0.53 & 1 \\
\hline
\end{tabular}

Table 5 Spearman correlation coefficient matrix calculated with Pareto-optimal solutions of (41)

\begin{tabular}{lllllllll}
\hline Objective & $-F_{\text {gas }}$ & $F_{\text {dhc }}$ & $F_{\text {ele1 }}$ & $F_{\text {ele2 }}$ & $F_{\text {ele3 }}$ & $F_{\text {ele4 }}$ & $F_{\text {ele5 }}$ \\
\hline$-F_{\text {gas }}$ & 1 & -0.94 & 0.02 & -0.02 & -0.01 & 0.01 & -0.06 \\
$F_{\text {dhc }}$ & -0.94 & 1 & -0.03 & -0.02 & -0.04 & 0.01 & -0.03 \\
$F_{\text {ele1 }}$ & -0.02 & -0.03 & 1 & 0.79 & 0.94 & -0.83 & -0.24 & -0.01 \\
$F_{\text {ele2 }}$ & -0.02 & -0.02 & 0.79 & 1 & 0.56 & -0.50 & -0.43 \\
$F_{\text {ele3 }}$ & -0.01 & -0.04 & 0.94 & 0.56 & 1 & -0.88 & -0.13 \\
$F_{\text {ele4 }}$ & 0.01 & 0.01 & -0.83 & -0.50 & -0.88 & 1 & -0.09 \\
$F_{\text {ele5 }}$ & -0.06 & -0.03 & -0.24 & -0.43 & -0.13 & 0.02 & 0.02 \\
$F_{\text {ele6 }}$ & -0.01 & 0.01 & -0.08 & -0.09 & -0.07 & 0.06 & 0.06 \\
$\sum R^{N}$ & -0.05 & -0.04 & 1.57 & 1.29 & 1.37 & -1.11 & 0.02 \\
\hline
\end{tabular}


divide the four objectives into two conflicting groups, which are displayed as follows:

$$
\begin{aligned}
& \min \left(-F_{\text {gas }}+F_{\text {ele5 }}, F_{\text {dhc }}+F_{\mathrm{a}}\right) \\
& \quad \text { s.t. } F_{\text {ele } 6} \leq 1 \\
& \min \left(-F_{\text {gas }}+F_{\mathrm{a}}, F_{\text {dhc }}+F_{\text {ele5 }}\right) \\
& \quad \text { s.t. } F_{\text {ele6 }} \leq 1
\end{aligned}
$$

The bi-objective formulations in (43) and (44), are optimized and the quality of the obtained solutions is visualized in the original objective space, as shown in Fig. 5 with yellow lines and green lines, respectively. It is clear that both versions can further improve the quality of Pareto-optimal solutions. Nevertheless, the problem formulation of (43) outperforms the other one, then (43) is selected as the final formulation of the MaOP established in this paper. The quality of its Pareto-optimal solutions can also be illustrated in two dimensions, as shown in Fig. 6.

The MGSO-ACL is applicable to optimize many objectives simultaneously whereas many other Pareto based multi-objective algorithms are only suitable for

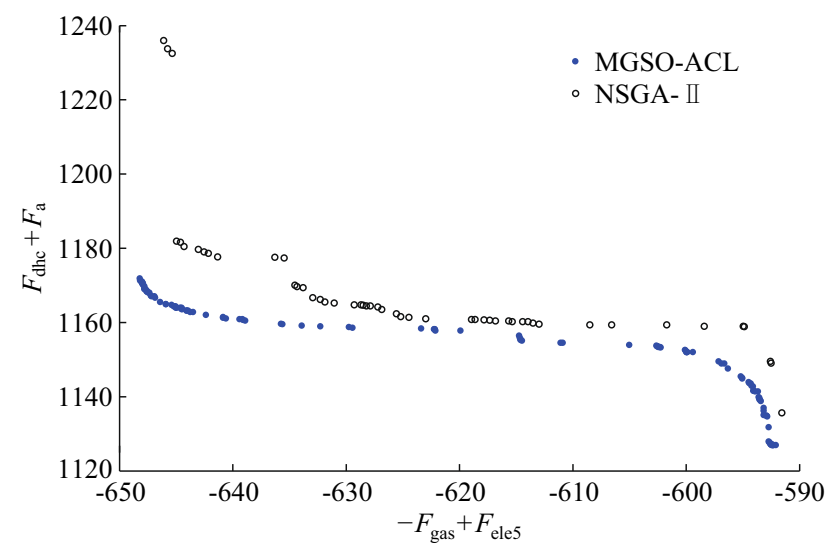

Fig. 6 Pareto-optimal fronts obtained by MGSO-ACL and NSGA-II with final objective set in (43) optimizing two or three objectives. By using our IOR approach, the final problem formulation is an essentially biobjective optimization problem which can also be solved by other Pareto based multi-objective algorithms, then we apply the popular multi-objective processing method, the elitist non-dominated sorting genetic algorithm (NSGA-II), to solve the bi-objective optimization model of the IES in (43). The results obtained by the NSGA-II with the same population size and iterations as MGSO-ACL are utilized as a comparison with those obtained by the MGSO-ACL. As shown in Fig. 6, it is obvious that the solutions obtained by MGSO-ACL all dominate those of the NSGA-II, indicating the better performance of MGSO-ACL in searching for more superior solutions, compared with NSGA-II.

In addition, it can be observed that the Pareto-optimal front of the bi-objective formulation is nonconvex, therefore, the front can not be obtained by using the weighting method [33]. Since the number of solutions has been reduced to a great extent and the corresponding objective values of the Pareto-optimal solutions are plotted intuitively in Fig. 6, it would be more efficient and convenient for the decision maker to select a preferable solution compared with optimizing eight objectives simultaneously.

In order to further compare the quality of solutions obtained by the original objective set in (36) and the final objective set in (43), the statistic data of their Pareto-optimal fronts and the selected dispatch solutions are listed in Table 7, where SS denotes the solution which is selected by the decision making method named improved entropy weight method. From this table, it can be seen that the original objective set only performs sightly better than the final objective set on $F_{\text {ele4 }}$ which is removed and $F_{\text {ele6 }}$ which is treated as a constraint. The results verify that our IOR approach is quite efficient in searching high quality solutions for the proposed many-objective optimization model, thus it will benefit the coordinated operation of the

\begin{tabular}{|c|c|c|c|c|c|c|c|c|c|c|}
\hline \multirow[t]{2}{*}{ Objective } & \multicolumn{5}{|c|}{ Original Pareto-optimal set } & \multicolumn{5}{|c|}{ Final Pareto-optimal set } \\
\hline & Min & Max & Mean & STD & SS & Min & Max & Mean & STD & SS \\
\hline$-F_{\text {gas }}$ & -647.37 & -504.53 & -600.55 & 25.915 & -633.51 & -648.76 & -592.87 & -620.80 & 23.3305 & -644.51 \\
\hline$F_{\mathrm{dhc}}$ & 602.60 & 700.11 & 634.00 & 18.141 & 639.33 & 602.24 & 645.76 & 628.58 & 13.483 & 637.90 \\
\hline$F_{\text {ele } 1}$ & 519.93 & 722.88 & 539.25 & 13.400 & 529.22 & 518.93 & 522.74 & 519.29 & 0.4076 & 519.14 \\
\hline$F_{\text {ele2 }}$ & 5.6990 & 26.054 & 8.2673 & 2.1269 & 7.5196 & 5.5144 & 6.7627 & 5.6764 & 0.1299 & 5.6502 \\
\hline$F_{\text {ele3 }}$ & 0.1677 & 0.2604 & 0.1721 & 0.0045 & 0.1689 & 0.1676 & 0.1683 & 0.1679 & 0.0002 & 0.0456 \\
\hline$F_{\text {ele } 4}$ & 0.0430 & 0.0636 & 0.0446 & 0.0016 & 0.0443 & 0.0449 & 0.0466 & 0.0457 & 0.0003 & 0.0456 \\
\hline$F_{\text {ele5 }}$ & 0.2800 & 2.9821 & 1.0448 & 0.4006 & 1.2203 & 0.4839 & 1.0589 & 0.7798 & 0.0969 & 0.7610 \\
\hline$F_{\text {ele6 }}$ & 0.9846 & 0.9877 & 0.9858 & 0.0005 & 0.9848 & 0.9862 & 0.9867 & 0.9864 & 0.0001 & 0.9863 \\
\hline
\end{tabular}
IES from the viewpoints of economy, security and environmental protection.

Table 7 Statistic data of original and final Pareto-optimal fronts and their selected solutions 


\section{Conclusion}

A many-objective optimization model has been developed to optimally coordinate the operation of the IES with electricity network, gas network and DHCs embedded. In order to improve the performance and applicability of many-objective optimization in tackling this model, we has proposed an objective reduction approach named IOR.

The simulation studies on a test IES show that during the objective reduction process, the fuel cost, power loss, and $\mathrm{NO}_{x}$ emission of electricity network are aggregated as one objective since they are in harmony with each other; the voltage stability index is transformed into a constraint as its values vary little during the optimization process; and the objective of $\mathrm{SO}_{2}$ emission is removed for the reason that the values of other objectives can be declined while its own values increase little. Finally, the proposed MaOP is reduced to be a bi-objective formulation with two conflicting groups of objectives. The simulation results have verified that the IOR approach is able to obtain better solutions to coordinate different objectives of the IES and alleviate the burden of the decision maker.

Finally, we would like to mention that the many-objective optimization method proposed in this paper, is actually a generic approach, and can be extended to other coordinated planning and operation issues of IES.

Acknowledgements This work was supported by the State Key Program of National Natural Science Foundation of China (No. 51437006) and Guangdong Innovative Research Team Program (No. 201001N0104744201).

Open Access This article is distributed under the terms of the Creative Commons Attribution 4.0 International License (http:// creativecommons.org/licenses/by/4.0/), which permits unrestricted use, distribution, and reproduction in any medium, provided you give appropriate credit to the original author(s) and the source, provide a link to the Creative Commons license, and indicate if changes were made.

\section{References}

[[1] Yannopoulos SI, Lyberatos G, Theodossiou N et al (2015) Evolution of water lifting devices (pumps) over the centuries worldwide. Water 7(9):5031-5060

[2] Qadrdan M, Wu J, Jenkins N et al (2014) Multi-stage flexible expansion co-planning under uncertainties in a combined electricity and gas market. IEEE Trans Sustain Energ 5(1):128-138

[3] Birol F (2010) World energy outlook 2010. International Energy Agency, Paris

[4] Wu T, Yang Q, Bao Z et al (2013) Coordinated energy dispatching in microgrid with wind power generation and plug-in electric vehicles. IEEE Trans Smart Grid 4(3):1453-1463

[5] Jing ZX, Jiang XS, Wu QH et al (2014) Modelling and optimal operation of a small-scale integrated energy based district heating and cooling system. Energy 73:399-415
[6] Wang H, Yin W, Abdollahi E et al (2015) Modelling and optimization of CHP based district heating system with renewable energy production and energy storage. Appl Energ 159:401-421

[7] Shahidehpour M, Fu Y, Wiedman T (2005) Impact of natural gas infrastructure on electric power systems. Proc IEEE 93(5):1042-1056

[8] Li T, Eremia M, Shahidehpour M (2008) Interdependency of natural gas network and power system security. IEEE Trans Power Syst 23(4):1817-1824

[9] Hu Y, Bie Z, Ding T et al (2015) An NSGA-II based multiobjective optimization for combined gas and electricity network expansion planning. Appl Energ 167:280-293

[10] Liu C, Shahidehpour M, Wang J (2011) Coordinated scheduling of electricity and natural gas infrastructures with a transient model for natural gas flow. Chaos Interdiscip J Nonlinear Sci 21(2):1-12

[11] Bai LQ, Li FX, Cui HT et al (2015) Interval optimization based operating strategy for gas-electricity integrated energy systems considering demand response and wind uncertainty. Appl Energ 167:270-279

[12] Zheng JH, Wu QH, Jing ZX (2016) Coordinated scheduling strategy to optimize conflicting benefits for daily operation of integrated electricity and gas networks. Appl Energ. doi:10. 1016/j.apenergy.2016.08.146

[13] Wu LH, Wang YN, Yuan XF et al (2010) Environmental/economic power dispatch problem using multi-objective differential evolution algorithm. Electr Power Syst Res 80(9):1171-1181

[14] Guo CX, Zhan JP, Wu QH (2012) Dynamic economic emission dispatch based on group search optimizer with multiple producers. Electr Power Syst Res 86:8-16

[15] Fleming PJ, Purshouse RC, Lygoe RJ (2005) Many-objective optimization: an engineering design perspective. In: Proceedings of the 3rd international conference on evolutionary multi-criterion optimization (EMO 2005). Lecture Notes in Computer Science, vol 3410. Springer, Heidelberg, pp 14-32

[16] Praditwong K, Yao X (2007) How well do multi-objective evolutionary algorithms scale to large problems. In: Proceedings of the IEEE congress on evolutionary computation (CEC 2007). IEEE, Singapore, pp 3959-3966

[17] Zheng JH, Chen JJ, Wu QH et al (2015) Multi-objective optimization and decision making for power dispatch of a largescale integrated energy system with distributed DHCs embedded. Appl Energ 154:369-379

[18] Deb K, Saxena D (2006) Searching for Pareto-optimal solutions through dimensionality reduction for certain large-dimensional multi-objective optimization problems. In: Proceedings of the world congress on computational intelligence (WCCI 2006), IEEE Computer Society Press, Los Alamitos, pp 3352-3360

[19] Dai C, Wang Y, Hu L (2015) An improved $\alpha$-dominance strategy for many-objective optimization problems. Soft Comput 20(3):1105-1111

[20] Ma X, Qi Y, Li L et al (2014) MOEA/D with uniform decomposition measurement for many-objective problems. Soft Comput 18(12):2541-2564

[21] Wang H, Yao X (2015) Objective reduction based on nonlinear correlation information entropy. Soft Comput 14(5):1-15

[22] de Freitas ARR, Fleming PJ, Guimarães FG (2015) Aggregation trees for visualization and dimension reduction in many-objective optimization. Inf Sci 298:288-314

[23] Silva R, Salimi A, Li M et al (2016) Visualization and analysis of tradeoffs in many-objective optimization: a case study on the interior permanent magnet motor design. IEEE Trans Magn 52(3): $1-4$

[24] Richardson AM (2015) Nonparametric statistics: a step-by-step approach. Int Stat Rev 83(1):163-164 
[25] Zhang X, Shahidehpour M, Alabdulwahab A et al (2016) Hourly electricity demand response in the stochastic day-ahead scheduling of coordinated electricity and natural gas networks. IEEE Trans Power Syst 31(1):592-601

[26] Qiu J, Dong ZY, Zhao JH et al (2015) Multi-stage flexible expansion co-planning under uncertainties in a combined electricity and gas market. IEEE Trans Power Syst 30(4):2119-2129

[27] An S, Li Q, Gedra TW (2003) Natural gas and electricity optimal power flow. In: Proceedings of the 2003 IEEE PES transmission and distribution conference, Dallas, Texas, 7-12 September 2003, pp 138-143

[28] Varadarajan M, Swarup KS (2008) Solving multi-objective optimal power flow using differential evolution. IET Gener Transm Distrib 2(5):720-730

[29] Niknam T, Narimani MR et al (2012) Improved particle swarm optimisation for multi-objective optimal power flow considering the cost, loss, emission and voltage stability index. IET Gener Transm Distrib 6(6):515-527

[30] Auger A, Hansen N (2012) Tutorial CMA-ES: evolution strategies and covariance matrix adaptation. In: GECCO (Companion), pp 827-848

[31] Yang XS (2010) Firefly algorithm, levy flights and global optimization. In: Bramer M, Ellis R, Petridis M (eds) Research and development in intelligent systems XXVI. Springer, London, pp 209-218

[32] Martínez-Mares A, Fuerte-Esquivel CR (2012) A unified gas and power flow analysis in natural gas and electricity coupled networks. IEEE Trans Power Syst 27(4):2156-2166

[33] Giagkiozis I, Fleming PJ (2015) Methods for multi-objective optimization: an analysis. Inf Sci 293:338-350

Y. N. KOU received the B.E. degree in Electrical Engineering from Huazhong University of Science and Technology, Wuhan, China, in 2014. She is currently pursuing the M.S. degree at the same area in South China University of Technology, Guangzhou, China. Her research interests include stochastic optimization for power system considering the integration of renewable energy, multi-objective optimization and its application on integrated energy systems.

J. H. ZHENG received his B.E. degree in Electrical Engineering from Huazhong University of Science and Technology, Wuhan,
China, in 2012. He is currently pursuing the Ph.D degree at the same area in South China University of Technology, Guangzhou, China. His research interests include the area of optimization, decision making and their applications on integrated energy systems.

Zhigang LI received the B.S and Ph.D degrees in electrical engineering from Tsinghua University, Beijing, China, in 2011 and 2016, respectively. He is currently an associate professor with the School of Electric Power Engineering, South China University of Technology, Guangzhou, China. He was a visiting scholar at the Illinois Institute of Technology, Chicago, IL, USA, and the Argonne National Laboratory, Argonne, IL, USA from 2014 to 2015. His research interests include renewable energy integration, power system optimization, and integrated energy systems.

Q. H. WU received the M.Sc. (Eng) degree in Electrical Engineering from Huazhong University of Science and Technology, Wuhan, China, in 1981. From 1981 to 1984, he was appointed Lecturer in Electrical Engineering in the University. He obtained a Ph.D degree in Electrical Engineering from The Queens University of Belfast (QUB), Belfast, U.K. in 1987. He worked as a Research Fellow and subsequently a Senior Research Fellow in QUB from 1987 to 1991. He joined the Department of Mathematical Sciences, Loughborough University, Loughborough, U.K. in 1991, as a Lecturer, subsequently he was appointed Senior Lecturer. In September, 1995, he joined The University of Liverpool, Liverpool, UK, to take up his appointment to the Chair of Electrical Engineering in the Department of Electrical Engineering and Electronics. Now he is with the School of Electric Power Engineering, South China University of Technology, Guangzhou, China, as a Distinguished Professor and the Director of Energy Research Institute of the University. Professor Wu has authored and coauthored more than 480 technical publications, including 240 journal papers, 20 book chapters and 3 research monographs published by Springer. He is a Fellow of IEEE, Fellow of IET, Chartered Engineer and Fellow of InstMC. His research interests include nonlinear adaptive control, mathematical morphology, evolutionary computation, power quality and power system control and operation. 